\title{
Non-Orthogonal Variable Spreading Factor-Time Multiplexing based Sensor Network Topology compared with Tree Routing and Enhance Tree Routing Protocol
}

\author{
Sharad \\ Assistant Professor, \\ BIT Engineering College, \\ Meerut, India.
}

\author{
Shailendra Mishra \\ Professor and Head, \\ CSE, BCTKEC, \\ Dwarahat, India.
}

\author{
Ashok Kumar Sharma \\ Professor and Head, CSE, \\ YMCA University, \\ Faridabad, India.
}

\author{
D. S. Chauhan \\ V. C, UTU, \\ Dehradun, India.
}

\begin{abstract}
Topology in a network plays an important role for effective and efficient data communication. It is infect the organization of network that depicts how the communication between two nodes will take place. Wireless sensor network is a collection of tiny self-organized sensor nodes. A sensor has a characteristic of sensing small data from the environment, process it and forward it to some collecting device. Since the device is too small, it has tiny power supply mechanism to support these activities. Energy for a bit transmission is dependent on the distance between sender and receiver. Therefore it is important to have a well designed topology for sensor network formation. Several protocols and schemes have been proposed by researchers for this purpose. This paper focuses on tree based topology architecture for sensor deployment and produces a comparison between a new proposed scheme and a few existing schemes.
\end{abstract}

Keywords: WSN, NOVSF-TM, topology, mobile sink

\section{INTRODUCTION}

A wireless ad-hoc sensor network [1][2] consists of a number of sensors spread over a large geographical area. Each sensor has wireless communication capability and some level of intelligence for signal processing [3] and networking of the data. Some examples of wireless ad hoc sensor networks are like Military sensor networks to detect and gain as much information as possible about enemy movements, explosions, and other phenomena of interest, Sensor networks to detect and characterize Chemical, Biological, Radiological, Nuclear, and Explosive (CBRNE) attacks and material, Sensor networks to detect and monitor environmental changes in plains, forests, oceans, etc. This suggests that wireless ad-hoc sensor networks offer certain capabilities and enhancements in operational efficiency in civilian applications as well as assist in the national effort to increase alertness to potential terrorist threats. Two ways to classify wireless ad-hoc sensor networks are whether or not the nodes are individually addressable, and whether the data in the network is aggregated. The sensor nodes in a parking lot network as an example should be individually addressable, so that one can determine the locations of all the free spaces. This application shows that it may be necessary to broadcast a message to all the nodes in the network. If one wants to determine the temperature in a corner of a room, then addressability may not be so important. Any node in the given region can respond. The ability of the sensor network to aggregate the data collected can greatly reduce the number of messages that need to be transmitted across the network. A cluster of sensors spread over a large geographical region to sense pattern behaves differently. As they need to sense data from the environment, process them and forward them to nearby station either sink node or any other neighbor node. This involves either single hopping or multiple hopping. Therefore there is a need to organize the sensor network in such a way that the link of communication can be established. The tree type structure is best suited to the situation, as sink node can work as the root of the tree and sensors can behave as the child of the sink node. The sensors deployed randomly in an environment forms a hierarchical structure for data forwarding. The structure is identified as tree topology.

The paper is organized in following sections: section 2 describes the two common tree based protocol for WSNs, called TR and ETR, section 3 gives a brief overview of NOVSF-TM technology based tree protocol, and section 4 will show comparison of the tree topologies for the three protocols and finally section 5 conclude the work.

\section{THE TREE ROUTING (TR) AND ENHANCE TREE (ETR) ROUTING TOPOLOGIES}

Since the transmit power of a wireless radio is proportional to distance squared, a direct communication over long distance consumes more energy than multi-hop communication. Moreover in a large area of interest multihop transmission is the appropriate way of communication. Topology creation, therefore, is an essential function of multi-hop WSN and routing is the method built into the firmware of each sensor node for finding paths between source and destinations. The elementary method of sensor network construction is to start with a root node (usually sink) and expand as new nodes join as child nodes. Each node can have multiple children but only one parent. The resultant network structure is like a tree as depicted in Figure 1. In Figure 1, nodes A, B and C are the child nodes of 
Sink/root node. Both sink and $\mathrm{C}$ are the ancestors of node $\mathrm{E}$ and $\mathrm{F}$ while all nodes except Sink are descendants' nodes. Tree routing [4] is well suited for such tree topology. The inter-node communication is restricted to parent-child links only. By relying solely on the parent-child links, TR eliminates path searching and updating complexities. TR is suitable for networks consisting of small-memory, lowpower and low-complexity lightweight nodes.

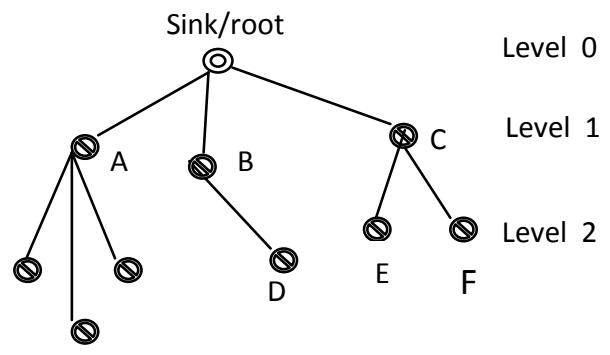

Figure 1: Tree Routing Structure

The main drawback of TR is the increased hop-counts as compared with other path search protocols. TR does not utilized neighbor table fully. A neighbor table records information such as addresses of nodes within the radio range, information of parent and child nodes etc. The neighbor table is created when the node joins a parent node. Enhance Tree Topology [4] uses the links to other one-hop neighbors for routing, if it is found to be shorter (in terms of hop count) than the tree path.

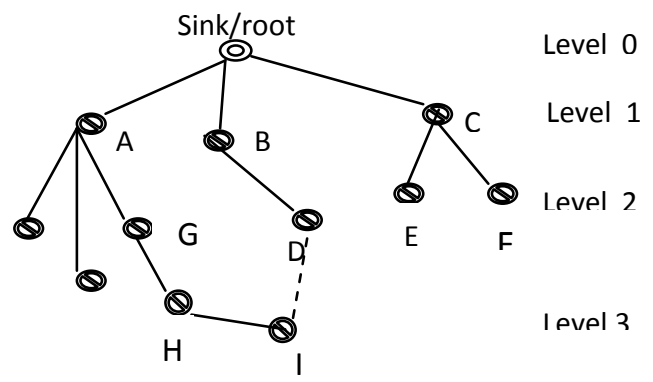

Figure 2: ETR Tree Structure

It uses minimum storage and computing cost to identify new paths by utilizing the address structure. It takes advantage of neighbor table to improve performance of TR protocol. Figure 2 shows the architecture of ETR Protocol. Here the node I will select the path (I, D, B to Sink, having hop-count 3) instead of the traditional path (I, H, G, A to Sink, having hop-count 4). ETR topology is thus found to be more effective for data communication than the TR topology. However additional overhead for neighbor discovery occurs when the number of sensors increases.

\section{THE NOVSF-TM TECHNOLOGY BASED TREE TOPOLOGY}

The proposed tree topology is based on addressing scheme derived from Non-blocking Orthogonal Variable Spreading Factor with Time Multiplexing (NOVSF-TM) [5] technique, which uses a spreading factor (SF-8) to generate non blocking orthogonal codes assigned to the mobile sink nodes. The mobile sink nodes are positioned at the centroid location of the polygon, logically created by joining the extreme sensors as coordinates, in the region where sensors are deployed. Figure 3 shows the topology for the NOVSF-TM based tree network with one mobile sink and a fixed sink node. Overall four mobile sink can be positioned in the region with SF-8[5]. Each sink is capable of supporting 128 sensors. The protocol thus, with SF-8 can support a maximum of 512 sensors (see Table 1).

Table 1: Code Assignment and Sensors Supported in NOVSF-TM based Tree Topology

\begin{tabular}{|c|c|c|c|c|c|c|}
\hline 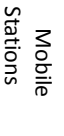 & $\begin{array}{l}2 \\
\stackrel{2}{0} \\
\stackrel{0}{0} \\
\stackrel{0}{\infty}\end{array}$ & 渵 & 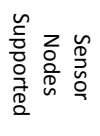 & 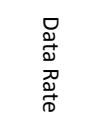 & 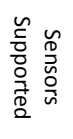 & 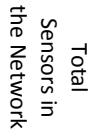 \\
\hline \multirow{2}{*}{ MS 1} & 11111111 & 8 & 64 & $64 * 8=512$ & $64+64$ & \multirow{9}{*}{$\begin{array}{l}\stackrel{\leftrightarrow}{N} \\
\infty \\
* \\
\stackrel{*}{*} \\
\stackrel{N}{N}\end{array}$} \\
\hline & $1111-1-1-1-1$ & 8 & 64 & $64 * 8=512$ & $=128$ & \\
\hline \multirow[b]{2}{*}{ MS 2} & 11-1-1 11-1-1 & 8 & 64 & $64 * 8=512$ & $64+64$ & \\
\hline & 11-1-1 -1-111 & 8 & 64 & $64 * 8=512$ & $=128$ & \\
\hline \multirow[b]{2}{*}{ MS 3} & 1-11-1 1-11-1 & 8 & 64 & $64 * 8=512$ & $64+64$ & \\
\hline & $1-11-1-11-11$ & 8 & 64 & $64 * 8=512$ & $=128$ & \\
\hline \multirow{3}{*}{ MS 4} & 1-1-11 1-1-11 & 8 & 64 & $64 * 8=512$ & $64+64$ & \\
\hline & & & & & & \\
\hline & $1-1-11-111-1$ & 8 & 64 & $64 * 8=512$ & $=128$ & \\
\hline
\end{tabular}

The mobile sink reduces the hop-count significantly as compared to ETR and TR protocols. The simulation results also shows noticeable differences in terms of topology creation and hop-count while transmission. Figure 4 below shows the NOVSF code assignment to the root node and the mobile sink nodes. The orthogonal nodes are non-blocking as time multiplexing is implemented in it.

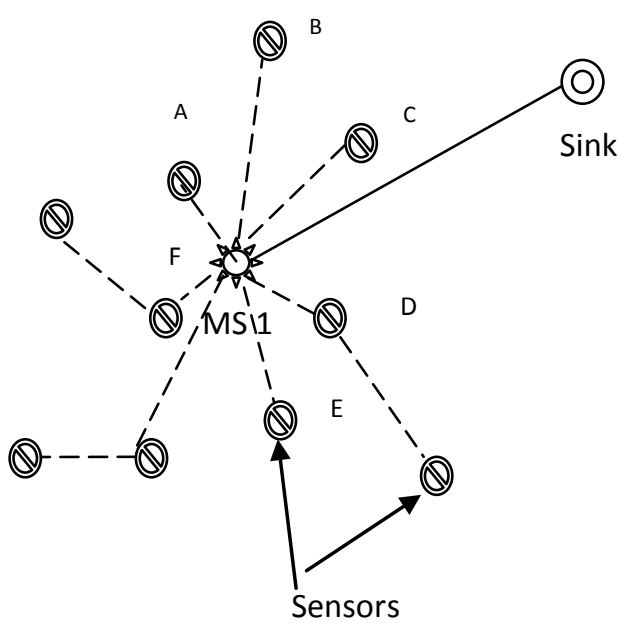

Figure 3: NOVSF-TM Based Tree topology with one mobile sink (MS1) and a fix sink node 


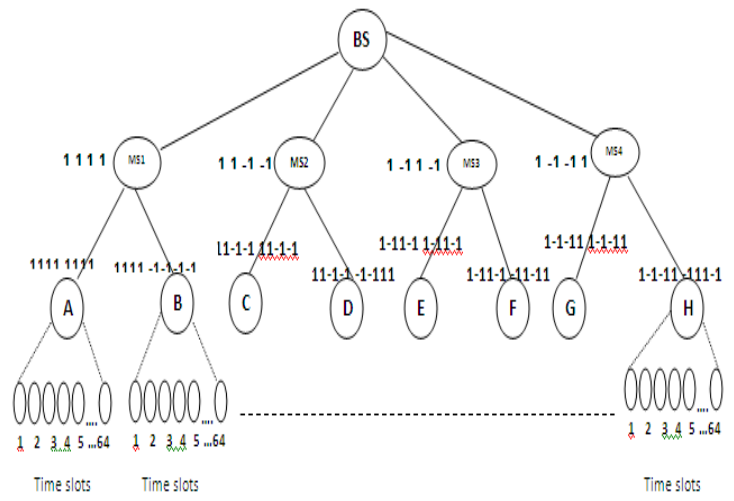

Figure 4: NOVSF-TM Based Tree Architecture

In Figure 4 the BS is the base station (sink) and MS (1-4) are the mobile base stations. A (1111 1111), B (1111 -1-1-1-1) Time multiplexed code assigned by MS1. C (11-1-1 11-1-1), D (11-1-1 -1-111) Time multiplexed code assigned by MS2. E (1-11-1 1-11-1), F (1-11-1 -11-11) Time multiplexed code assigned by MS3. G (1-1-11 1-1-11), H (1-1-11 -111-1) Time multiplexed code assigned by MS4.

\section{TOPOLOGIES COMPARISON}

The network topology simulation is performed in MATLAB for the three protocols. The simulation has been performed in a fixed region of 500x500 for number of nodes 50, 60, 65 respectively. The topology graphs obtained are as:

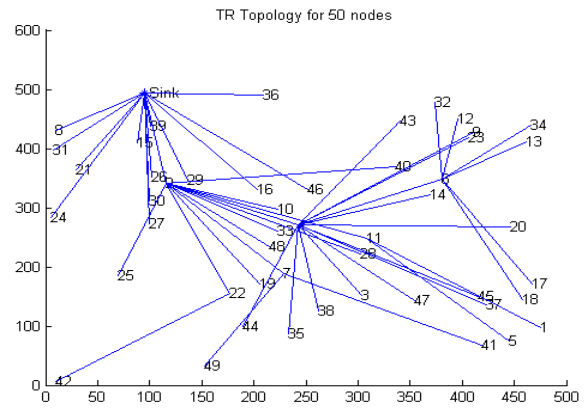

(a)

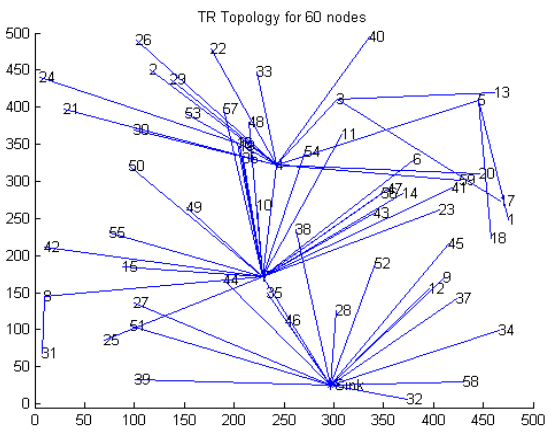

(b)

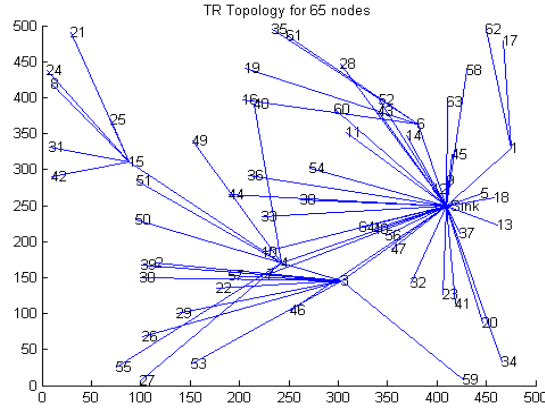

(c)

Figure 5: TR Topology for nodes $(50,60,65)[(a),(b),(c)$ respectively]

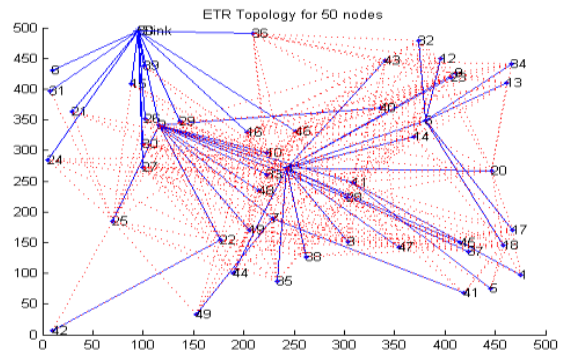

(d)

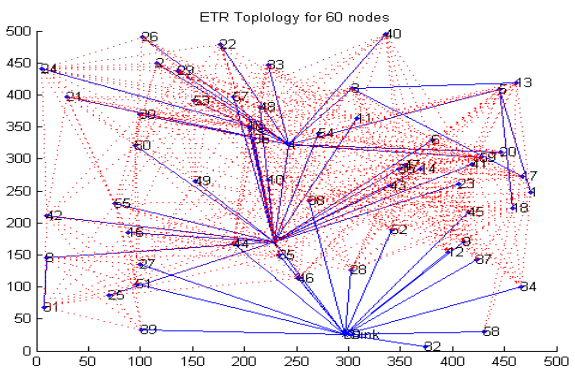

(e)

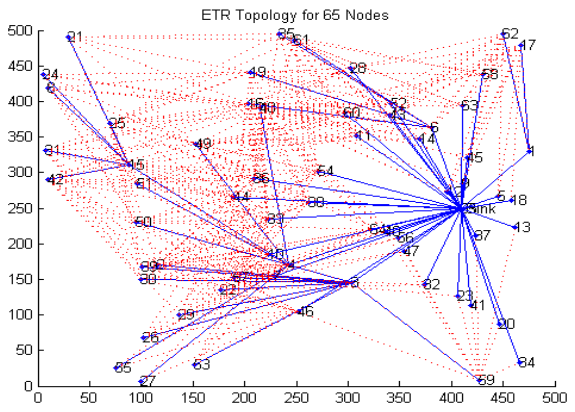

(f)

Figure 6: ETR Topology for nodes $(50,60,65)[(d),(e),(f)$ respectively] 


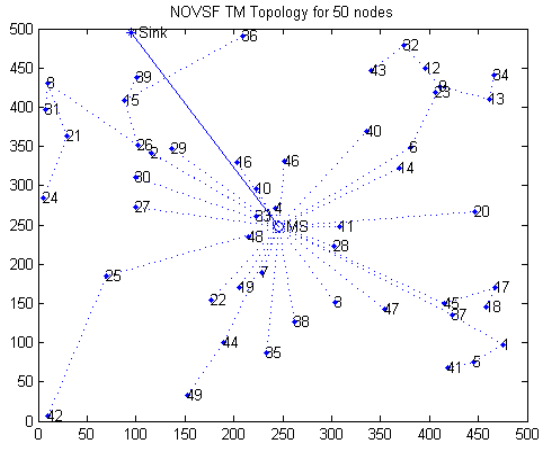

(g)

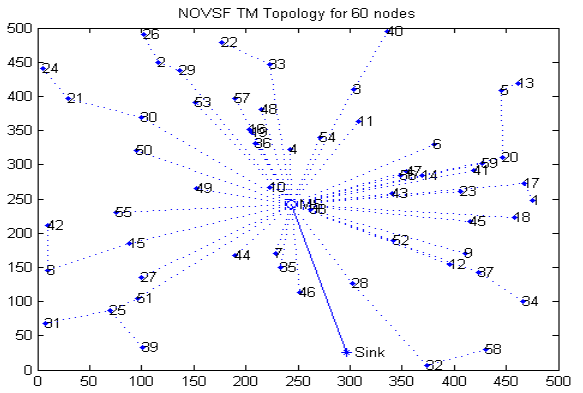

(h)

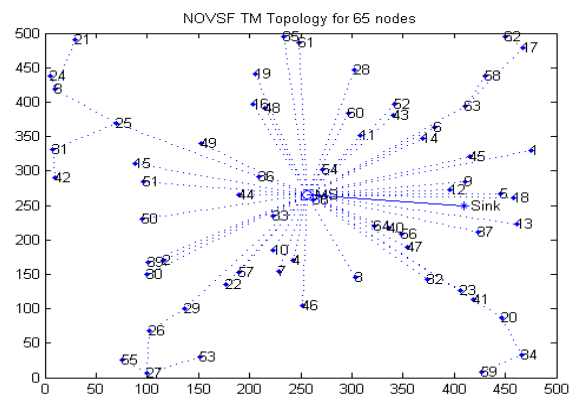

(i)

Figure 7: NOVSF-TM Based Tree Topology for nodes $(50,60,65)[(g),(h),(i)$ respectively $]$

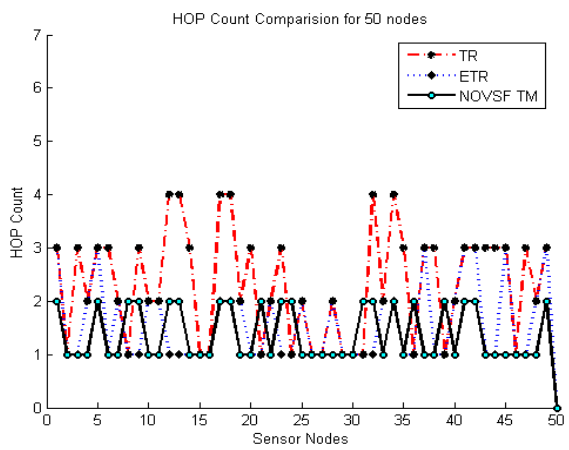

(j)

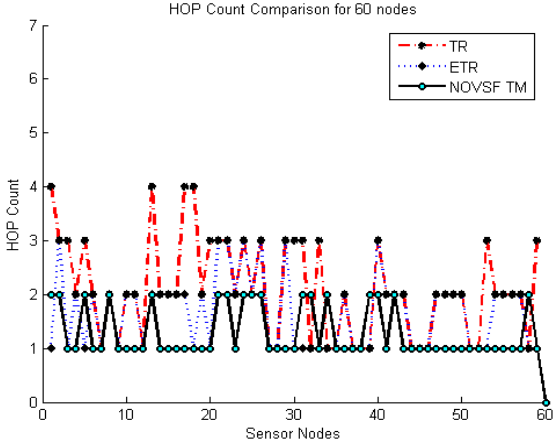

(k)

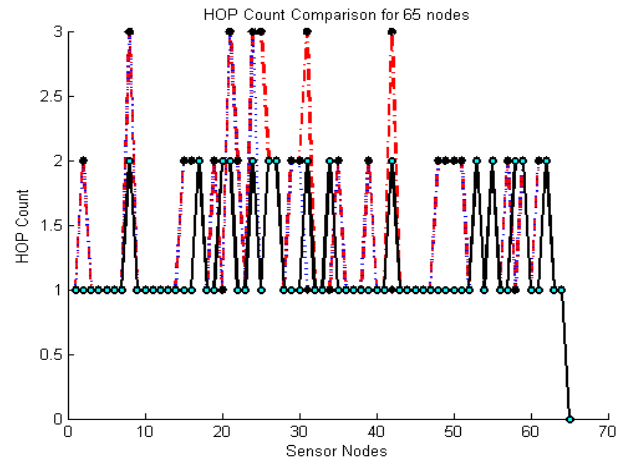

(1)

Figure 8: HOP Count Graph for nodes $(50,60,65)[(j)$, (k), (l) respectively]

It is evident from Figure 5 that TR protocol is well suited for small density networks and follows a fixed path for data transmission. The links become more complicated as the number of sensors increases. Figure 6 shows ETR protocol that considers the neighbor table for alternate shortest paths. The blue line in the graph is the traditional parent-child link created by TR protocol and the dotted red line are the alternative shortest path identified from the neighbor table. Figure 7 shows the NOVSF-TM based sensor network topology which shows a significant and noticeable difference in network topology as compared to the TR and ETR protocol topologies. Mobile sink in the network help in reducing excessive multi-hopping to single-hopping where possible. Thus, reduction in excessive multi-hoping enable transmission energy saving. Figure 8 depicts hop-count reduction graph for the three protocols. The red line shows TR protocol, blue shows ETR protocol and black line shows the NOVSF-TM based tree protocol.

\section{CONCLUSION}

It is evident from the simulation results obtained that for small sensor network deployment the TR topology is simple and best for both energy saving and small hop-counts. Moreover there is no need of flooding path search messages on the network as it follows strict parent-child links for data forwarding. This helps in reduction of traffic congestion in the network. For a large network the topology is not suitable 
because it leads to formation of a long parent-child links and increases excessive multi-hoping. The ETR topology on the other hand makes full utilization of neighbor table, containing information of nearby sensors, for shortest possible path selection. This topology thus reduces excessive multi-hoping and forward the data with shortest possible path. Again for a dense sensor network the possibilities for multiple shortest paths exist. Therefore the topology and routing protocol suffers an overhead of neighbor discovery and path selection procedure. The NOVSF-TM based tree topology on the other hand is based on the spreading factor concept and orthogonal code generation. The root (sink) node determines the orthogonal codes and assigns them to the downward hierarchy. The introduction of mobile sinks in the dense sensor network helps in reduction of excessive multi-hoping. The sensors can directly send their data to the mobile sinks and mobile sink can further forward it to the fixed sink node. The spreading factor in the network determines the count of the mobile sink nodes that can be placed in the network and maximum capacity of the network. The NOVSF-TM based tree topology is observed to be more efficient and energy saving as compared to the TR and ETR tree topologies.

\section{REFERENCES}

[1] Ian Akyildiz, Weilian Su, Yogesh Sankarasubramaniam, and Erdal Cayirci, A Survey on Sensor Networks, IEEE Communications Magazine, pp. 102-104, 2002.

[2] V. Raghunathan, C. Schurghers, S. Park, M. Srivastava, Energy-aware wireless micro sensor networks, IEEE Signal Processing Magazine (2002) 40-50.

[3] F. Zhao, L. Guibas, Wireless Sensor Networks: An Information Processing Approach, Elsevier-Morgan Kaufmann, Boston, 2004

[4] Wanzhi Qiu, Efstratios Skafidas, Peng Hao, Enhanced tree routing for wireless sensor networks, Elsevier- Ad hoc Networks, pp. 638-650, 2009.

[5] Kiran Vadde and Hasan Cam, A Code Assignment Algorithm for Nonblocking OVSF Codes in WCDMA, Telecommunication Systems 25:3,4, 417-431, 2004, Kluwer Academic Publisher, Netherland 